\author{
Erminio PIVA*
}

\title{
Una nuova specie di Orotrechus Müller dei Colli Euganei (Veneto, Italia) (Coleoptera Carabidae Trechinae)
}

\begin{abstract}
Riassunto: Viene qui descritto Orotrechus frassanellicus n. sp., di ambienti ipogei dei Colli Euganei settentrionali (Veneto, Italia), nel territorio del comune di Rovolon (Padova). Affine ad O. euganeus Pace, 1974 (s.1.), se ne distingue particolarmente per la diversa forma del lobo mediano dell'edeago.
\end{abstract}

\begin{abstract}
A new species of Orotrechus Müller from Colli Euganei (Veneto, Italia) (Coleoptera Carabidae Trechinae)
Orotrechus frassanellicus, new species from hypogean environments in northern Colli Euganei (Veneto, Italy), in the municipality of Rovolon (Padova), is here described. It is very close to Orotrechus euganeus Pace, 1974 (s.1.), from which markedly differs for the shape of the median lobe of aedeagus.
\end{abstract}

Key words: Trechinae, Orotrechus, new species, Colli Euganei, Veneto, Italy.

\section{INTRODUZIONE}

Riesaminando numerosi reperti di Orotrechus Müller, 1913, provenienti da varie località dei Colli Euganei, ho potuto constatare la presenza di una nuova specie, descritta in questa nota, attribuita finora ad Orotrechus euganeus Pace, 1974: si tratta delle popolazioni più settentrionali del complesso euganeo, tutte comprese nel territorio del comune di Rovolon, in provincia di Padova.

Il materiale studiato è conservato nelle seguenti collezioni: Dante Bianco, Santorso, Vicenza (CBI); Luca Bodei, Brescia (CBO); Francesca Casa e Nicola Righetto, Schio, Vicenza (CCR); Achille Casale, Torino (CCA); Pier Mauro Giachino, Torino (CGI); Mario Grottolo, Brescia (CGR); Paolo Magrini, Firenze (CMA); Riccardo Monguzzi, Milano (CMR); Vittorino Monzini, San Giuliano Milanese, Milano (CMV); Museo Civico di Storia Naturale di Verona (CMU); Erminio Piva, Vicenza (CPI); Giuliano Trezzi, Sesto S. Giovanni, Milano (CTR).

\section{Orotrechus frassanellicus sp. n.}

Diagnosi. Un Orotrechus di medie dimensioni, strettamente affine ad $O$. euganeus s.1., ma distinto da questo e dalle altre specie congeneri per la forma del lobo mediano dell'edeago.
Località tipica. Veneto, Colli Euganei. Rovolon (PD); Buco del Tempietto (1399 V/PD), 46 m [GPS 4123].

Serie tipica. Holotypus $\widehat{\jmath}$ : Veneto, Colli Euganei. Rovolon (PD), Buco del Tempietto (1399 V/PD), 46 m [GPS 4123], 23.XI.2017, E. Piva leg. (CPI). Paratipi, 225 exx. (117 ડำ 108 우): Veneto, Colli Euganei. Rovolon (PD), Buco del Tempietto (1399 V/PD), $46 \mathrm{~m}$ [GPS 4123], 5 ๙ิ 1 ㅇ (CBI); ibidem, 1 ๆ (CMU); ibidem, 15 วิ స̂ 19 우 (CPI). Veneto, Colli Euganei. Rovolon (PD), Busa de l'Orso (1398 V/PD), 43 m [GPS 4139], 4 취 4 우 (CBI); ibidem, 1 ㅅ 1 ㅇ (CBO); ibi-

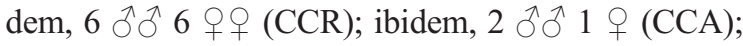
ibidem, 1 ㅅ 2 우 (CGI); ibidem, 8 ภㅅ 6 우 (CGR); ibidem, 3 우 (CMA); ibidem, 26 ㅊํ 12 우 (CMR); ibidem, 2 ઈิ 1 q (CMU); ibidem, 1 q (CMV); ibi-

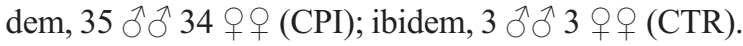
Veneto, Colli Euganei. Rovolon (PD), piccola cavità a nord-est del Monte Spinazzola e a sud della Fornace,

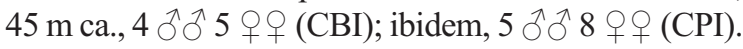

Derivatio nominis. Il nome specifico prende origine dal Parco di Frassanelle, nel cui comprensorio è situata la località tipica del nuovo taxon.

Descrizione dell'holotypus $\hat{\jmath}$. Corpo robusto (Fig. 1), lungo $4.43 \mathrm{~mm}$ (misurato dall'apice delle mandi-

*Erminio Piva, via G. Amendola 4, 36100 Vicenza (VI), Italia. E-mail: erminiopiva1@virgilio.it 
bole all'apice elitrale), lucido, glabro, di colore testaceo, con appendici moderatamente allungate; tegumenti con microscultura finissima a maglie poligonali irregolari.

Capo lungo $0.67 \mathrm{~mm}$ (dal margine anteriore del clipeo al restringimento collare) e largo altrettanto. Occhi assenti, tempie poco convesse, glabre, ma con due brevi setole in posizione arretrata; solchi frontali completi e bene incisi fino alla seconda setola sopraorbitale, fortemente divergenti in addietro. Presenti due setole sopraorbitali per lato. Antenne lunghe $2.62 \mathrm{~mm}$ (rapporto lungh. antenne/lungh. corpo $=0.59$ ); mandibole robuste e allungate.

Protorace poco trasverso, lungo $0.73 \mathrm{~mm} \mathrm{e}$ largo $0.78 \mathrm{~mm}$ (rapporto lu/la: 0.94), a lati regolar-

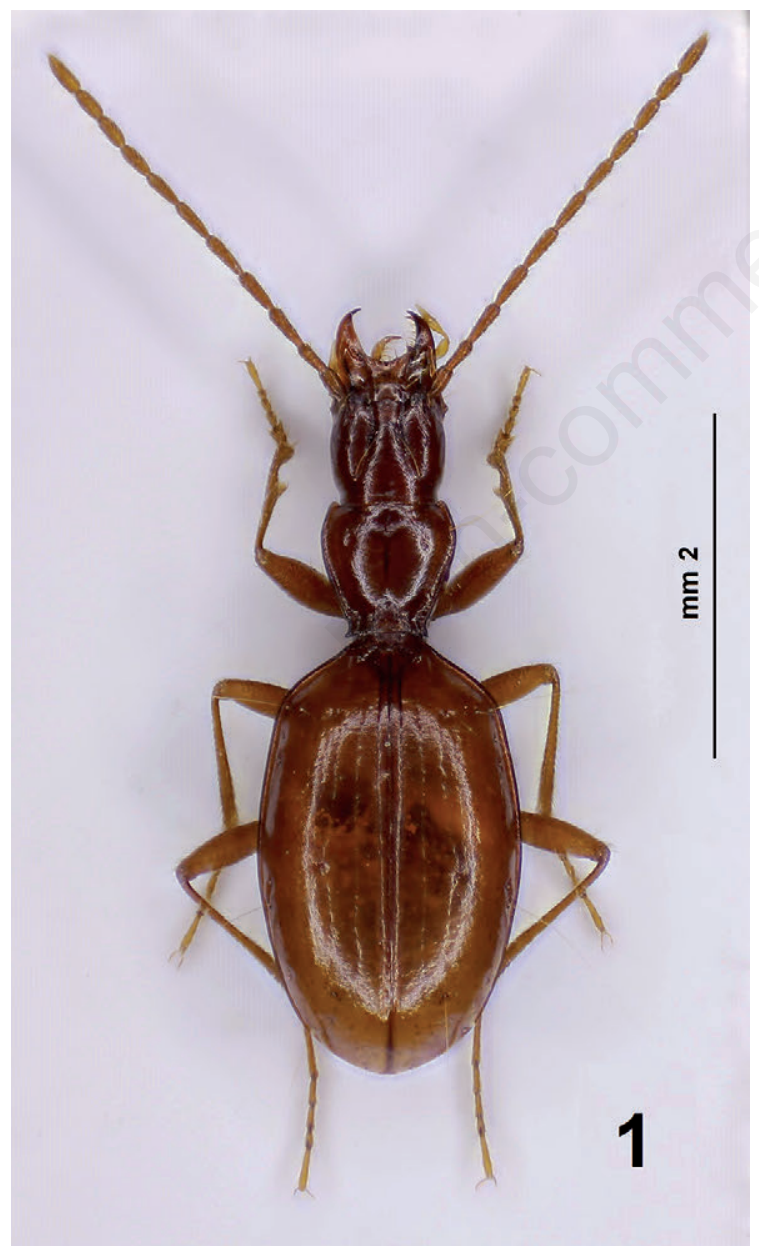

Fig. 1. Orotrechus frassanellicus sp. n., holotypus $\partial^{\lambda}$ : habitus. mente arrotondati e nettamente sinuati prima della base; angoli posteriori acuti e salienti all'esterno, angoli anteriori decisamente sporgenti in avanti e strettamente arrotondati. Doccia marginale stretta e regolare. Setole pronotali anteriori di normale sviluppo, inserite circa nel quinto anteriore; setola basale dell'angolo posteriore destro breve ed esile, mancante nell'angolo a sinistra.

Elitre ellittiche, convesse, lunghe 1.61 volte la loro larghezza (la lunghezza misurata dal vertice del triangolo scutellare all'apice, la larghezza considerate insieme); omeri arrotondati ma bene evidenti, doccia laterale discretamente ampia, carena apicale breve. Stria suturale fortemente incisa, le strie seconda e terza gradatamente meno impresse, le altre svanite. Chetotassi: setola scutellare bene sviluppata; serie discale di due setole, inserite sulla terza stria: l'anteriore appena dopo il livello della prima della serie ombelicata, la posteriore prima del livello della quinta della serie ombelicata. Gruppo omerale con la prima setola più ravvicinata alla seconda che non la terza rispetto alla quarta; seconda e terza maggiormente distanziate tra loro. Gruppi mediano e apicale senza caratteristiche particolari; triangolo apicale completo.

Zampe con tibie e tarsi pubescenti; primo protarsomero dilatato e dentato internamente.

Apparato copulatore: edeago lungo $0.61 \mathrm{~mm}$ (misurato dalla base all'apice). In visione laterale (Fig. 5) il lobo mediano risulta snello e affusolato, con bulbo basale poco sviluppato; il margine ventrale, inizialmente rettilineo, piega decisamente verso il basso nella sua metà distale. In visione dorsale l'apice è di forma ogivale (Fig. 6). Parameri muniti entrambi di diciotto setole. Lamella copulatrice stretta e allungata, ritorta alle due estremità.

Descrizione dei paratipi e variabilità. Lunghezza del corpo (dall'apice delle mandibole all'apice elitrale):

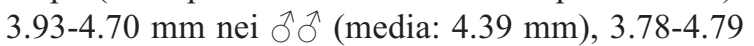
$\mathrm{mm}$ nelle 우 $ᄋ$ (media: $4.44 \mathrm{~mm}$ ). Il rapporto lunghezza antenne/lunghezza corpo è di 0.56-0.62 nei

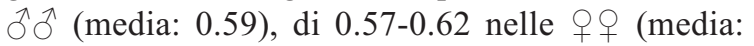

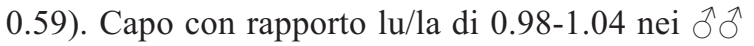
(media: 1.01), di 0.97-1.06 nelle 우우 (media: 1.01) (la lunghezza del capo misurata dal margine anteriore del clipeo al restringimento collare). Pronoto (Figg. 1214) (la cui lunghezza corrisponde alla linea mediana) con rapporto lu/la di 0.92-1.00 nei $\widehat{\partial} \widehat{\partial}$ (media: 0.95), di 0.92-0.97 nelle 웅 (media: 0.94); lati nettamente 

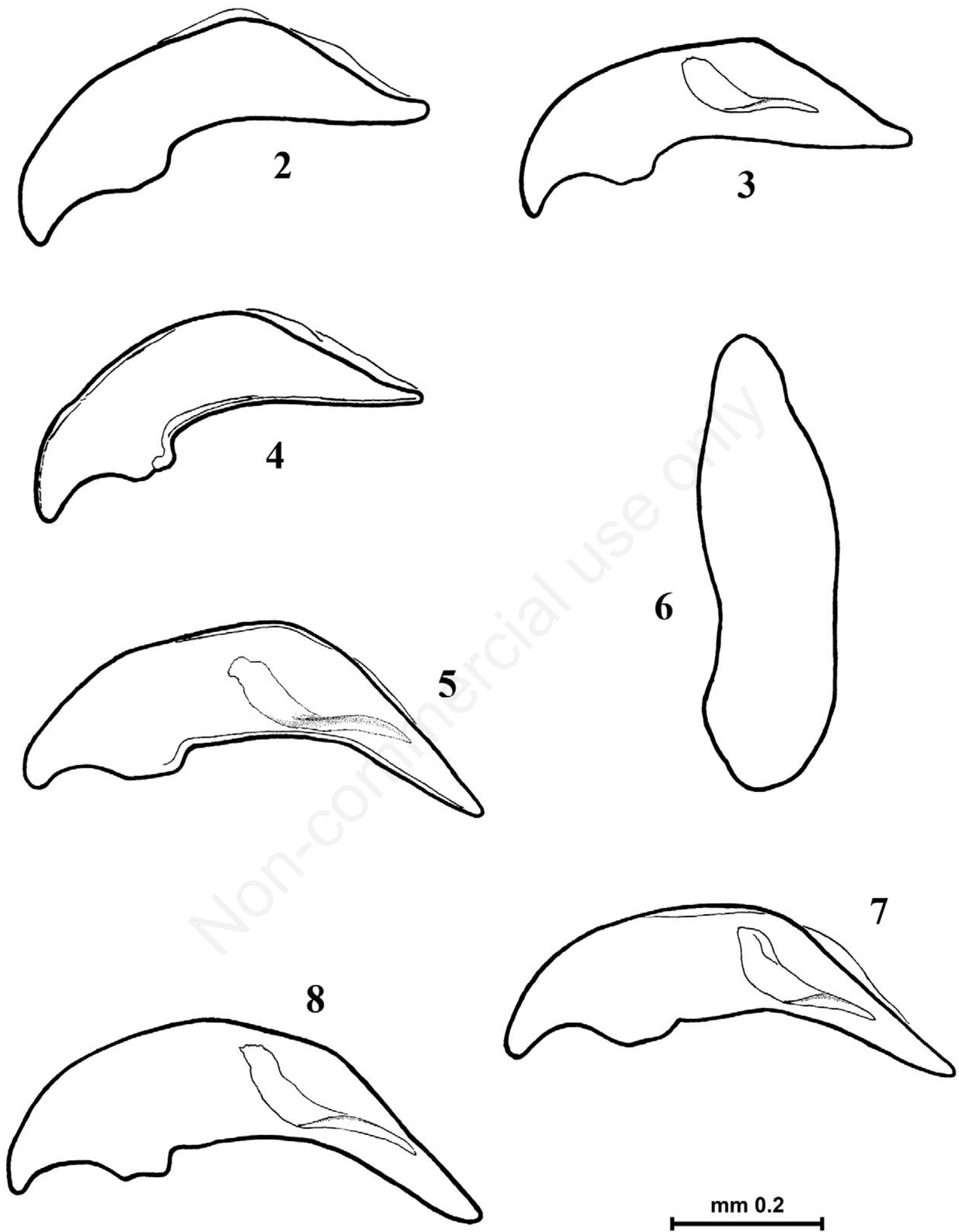

$\mathrm{mm} 0.2$

Figg. 2-8. Lobo mediano dell'edeago, in visione laterale (2-5, 7-8) e dorsale (6). Orotrechus euganeus euganeus, 2: paratypus (M. Ventolone, Arquà Petrarca); 3: esemplare di M. Fasolo, Cinto Euganeo. Orotrechus euganeus perettoi, 4: holotypus (da: Piva, 2014, modificato). Orotrechus frassanellicus sp. n., 5-6: holotypus; 7: paratypus di Busa de l'Orso, Rovolon; 8: paratypus della cavità a nord-est del M. Spinazzola, Rovolon. 
sinuati prima degli angoli posteriori, che sono acuti e sporgenti all'infuori. Angoli anteriori evidentemente protesi in avanti, tranne in un singolo caso. Setole basali normalmente presenti, raramente mancanti (probabilmente per caduta). Elitre con rapporto lunghezza/larghezza (considerate insieme) di 1.49-

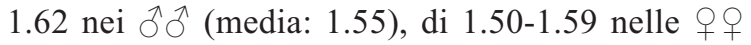
(media: 1.54). Chetotassi variabile: in particolare la posizione del primo poro della serie discale rispetto al secondo della serie ombelicata, spesso oltre il suo livello, ma talvolta prima di questo. Nei maschi l'apparato copulatore presenta il lobo mediano conforme a quello dell'olotipo (Figg. 7, 8); parameri muniti di quindici-ventidue setole. Lamella copulatrice stretta e allungata, ritorta alle estremità, da subtriangolare a subrettangolare.

Paratipi femmine con morfologia esterna simile ai maschi, ma con protarsi semplici. Armatura genitale con gonostili subtriangolari, strettamente arrotondati all'apice, muniti normalmente di due setole tergali, una delle quali molto robusta; due individui presentano un gonostilo con una terza setola tergale. Le fossette preapicali sono munite di due microsetole e di uno-due bastoncelli sensoriali, o prive di questi ultimi.

Note comparative. Ho potuto esaminare 15 esemplari (8 $\widehat{\jmath}, 7$ 우) di Orotrechus euganeus enganeus Pace, 1974: l'olotipo ${ }^{\Uparrow}$ (CMU), l'allotipo $\odot$ (CMU), 2 pa-

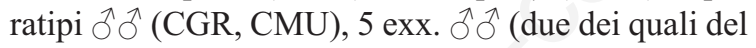
versante nord del M. Ventolone, Arquà Petrarca, in CPI; due della località Faedo, Cinto Euganeo, in CMR; uno del versante nord-orientale del M. Fasolo, Cinto Euganeo, in CPI) e 6 exx. 우우 (di cui due del versante nord del $\mathrm{M}$. Ventolone, Arquà Petrarca, in CPI; quattro del versante nord-orientale del M. Fasolo, Cinto Euganeo, in CBI e in CPI). I dati biometrici rilevati sono i seguenti: lunghezza corpo: $4.30-4.60 \mathrm{~mm}$ nei $\widehat{\partial} \widehat{\partial}$ (media: $4.38 \mathrm{~mm}$ ), $4.00-4.37 \mathrm{~mm}$ nelle $q$ 우 (media: $4.27 \mathrm{~mm}$ ). Il rapporto lunghezza antenne/lun-

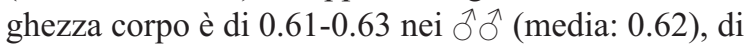
0.60-0.61 nelle 우우 (media: 0.60). Capo con rapporto

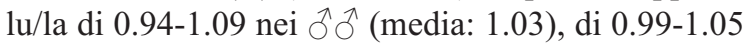
nelle 우우 (media: 1.02) (la lunghezza del capo misurata dal margine anteriore del clipeo al restringimento collare). Pronoto (la cui lunghezza corrisponde alla

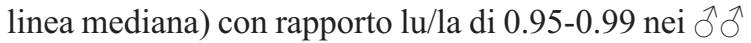
(media: 0.97), di 0.94-1.00 nelle 우 우 (media: 0.97); lati sinuati prima degli angoli posteriori, questi acuti e sporgenti all'infuori; angoli anteriori appena prominenti in avanti (Figg. 9, 10). Setole basali di norma presenti, raramente mancanti (per probabile caduta). Elitre con rapporto lu/la (considerate insieme) di 1.501.67; chetotassi variabile.

Nei maschi, edeago con lobo mediano di forma costante (Figg. 2, 3), parameri muniti di 14-20 setole. Nelle femmine, armatura genitale senza particolarità di rilievo.

Inoltre, ho riesaminato 41 esemplari della serie

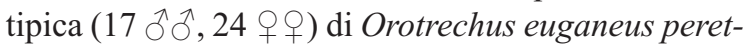
toi Piva, 2014: l'olotipo $\widehat{\partial}$ (Figg. 4, 11) (CPI) e 40 paratipi (CPI). I dati biometrici rilevati sono i seguenti: lunghezza corpo: 3.56-3.99 mm nei $\widehat{\partial} \widehat{\partial}$ (media: 3.77 $\mathrm{mm}$ ), 3.50-4.02 mm nelle 우우 (media: $3.74 \mathrm{~mm}$ ). Il rapporto lunghezza antenne/lunghezza corpo è di $0.57-0.60$ nei $\widehat{\partial} \widehat{\partial}$ (media: 0.58 ), di $0.55-0.61$ nelle 우우 (media: 0.58). Capo con rapporto lu/la di 0.93-0.99 nei $\widehat{\partial} \widehat{\partial}$ (media: 0.96), di 0.91-0.99 nelle 웅 (media: 0.96 ) (la lunghezza del capo misurata dal margine anteriore del clipeo al restringimento collare). Pronoto (la cui lunghezza corrisponde alla linea mediana) con rapporto lu/la di 0.88-0.96 nei $\widehat{\partial} \widehat{\widehat{O}}$ (media: 0.91), di 0.88-0.96 nelle 우우 (media: 0.90); lati sinuati prima degli angoli posteriori, che sono acuti e sporgenti all'infuori; angoli anteriori più o meno prominenti in avanti. Setole basali presenti, tranne in due casi, dove mancano per probabile caduta. Elitre con rapporto lu/la (considerate insieme) di 1.51-1.68. Nei maschi l'apparato copulatore presenta il lobo mediano conforme a quello dell'olotipo (Fig. 4); variabile il numero di setole dei parameri.

Dall'esame biometrico non si evidenziano valori differenziali significativi per distinguere la nuova specie, ma un particolare risulta sufficientemente costante (con una sola eccezione osservata), cioè la notevole espansione in avanti degli angoli anteriori del pronoto (Figg. 12-14). Resta sicuro, invece, il riconoscimento del nuovo taxon in base alla morfologia del lobo mediano dell'edeago (Figg. 5-8).

Distribuzione. La nuova specie proviene da tre ambienti ipogei: due di essi, il Buco del Tempietto (località tipica) e la Busa de l'Orso, sono tra le grotte più importanti dei Colli Euganei, la prima per essere l'unica a sviluppo verticale (profonda 16 metri), la seconda per avere la maggiore estensione (60 metri). Entrambe si aprono nel Parco di Frassanelle, tenuta dei conti Papafava, su fronti di cave abbandonate, affio- 

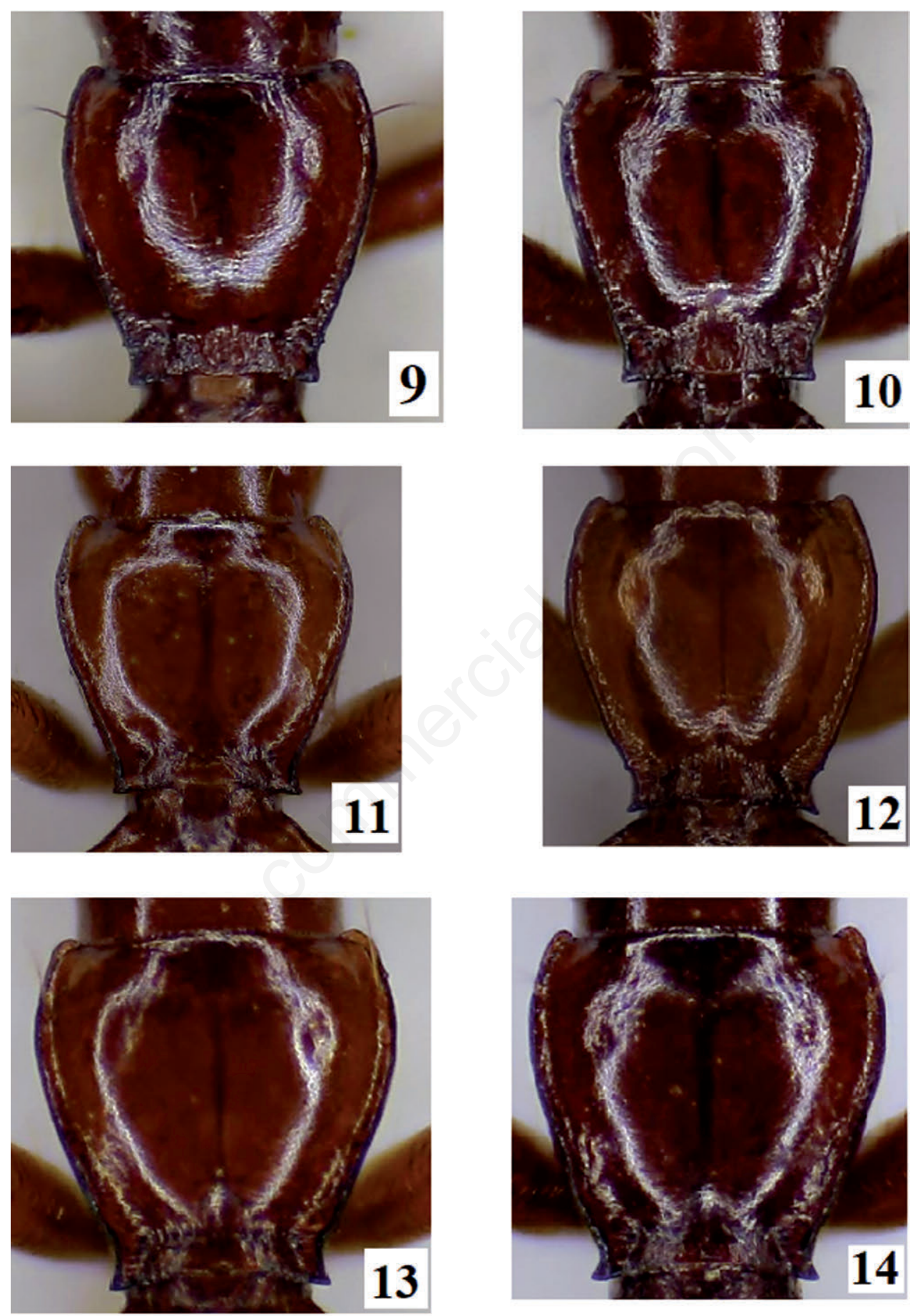

\section{mm 0.5}

Figg. 9-14. Pronoto. Orotrechus euganeus euganeus, 9: paratypus (M. Ventolone, Arquà Petrarca); 10: esemplare di M. Fasolo, Cinto Euganeo. Orotrechus euganeus perettoi, 11: holotypus. Orotrechus frassanellicus sp. n., 12: paratypus topotipico (Buco del Tempietto, Rovolon); 13: paratypus (Busa de l'Orso, Rovolon); 14: paratypus (cavità a nord-est del M. Spinazzola, Rovolon). 
ranti nella formazione sedimentaria della scaglia rossa, di età compresa tra il Cretaceo superiore e l'Eocene inferiore (Panajotti et al., 1979).

La terza stazione di rinvenimento è una modesta cavità sul versante nord-orientale del Monte Spinazzola, anch'essa situata nel territorio comunale di Rovolon.

Osservazioni. L'indiscussa somiglianza nella morfologia esterna delle due specie euganee ha generato inevitabilmente l'attribuzione di tutte le popolazioni all'unica entità fino ad allora nota per l'area, O. euganeus; l'esame dell'edeago, invece, non lascia dubbi sull'istituzione di una specie distinta per i reperti di Rovolon. Per quest'ultimo carattere $O$. frassanellicus sp. n. si differenzia da tutte le entità affini, distribuite nel settore sud-occidentale delle Prealpi Venete: $O$. vicentinus martinellii Daffner, 1987, del Monte Baldo; O. vicentinus iuccii Pomini, 1940 e O. vicentinus vicentinus (Gestro, 1907), dei Monti Lessini; O. fabianii (Gestro, 1900) e O. euganeus perettoi Piva, 2014, dei Monti Berici; O. euganeus euganeus Pace, 1974, dei Colli Euganei meridionali.

La notevole complessità del genere Orotrechus, che ha indotto molti studiosi a definire un gran numero di gruppi di specie (sintetizzati in Vigna Taglianti, 1981, 1982), è stata di recente confermata anche sulla base di indagini genetiche (Faille et al.,
2013); pur concordando sull'esigenza di un'opportuna revisione tassonomica, appare evidente che resta ancora molto lavoro da svolgere, soprattutto nella ridescrizione delle varie specie, sia riguardo alle loro affinità reciproche sotto il profilo filetico, sia alla loro attribuzione ai rispettivi raggruppamenti.

\section{RINGRAZIAMENTI}

Sono grato a tutti coloro che mi hanno permesso di esaminare il loro materiale: Dante Bianco, Luca Bodei, Francesca Casa, Achille Casale, Pier Mauro Giachino, Mario Grottolo, Leonardo Latella (per il Museo Civico di Storia Naturale di Verona), Paolo Magrini, Riccardo Monguzzi, Vittorino Monzini, Nicola Righetto, Giuliano Trezzi. Ringrazio, inoltre, Michele Gallo, Michele Miazzi, Renato Rosa ed Enrico Specchio, dipendenti del Parco Regionale dei Colli Euganei, per l'autorizzazione a campionare reperti, per motivi di studio, nel territorio del Parco. Sono grato ai conti Papafava (in particolare ad Alessandro), proprietari del fondo nel quale sono ubicate due delle grotte indagate, per l'autorizzazione a condurvi ricerche; ad Achille Casale e a Roberto Poggi, per i preziosi consigli. Infine, un ringraziamento al Club Speleologico Proteo di Vicenza, per il parziale contributo finanziario destinato alle ricerche biospeleologiche.

\section{BIBLIOGRAFIA}

DAFFner H., 1987 - Orotrechus martinellii spec. nov. vom Monte Baldo-Norditalien (Coleoptera, Carabidae, Trechinae). Nachrichtenblatt der Bayerischen Entomologen, 36 (2): 39-43.

Faille A., Casale A., Balke M., Ribera I., 2013 - A molecular phylogeny of Alpine subterranean Trechini (Coleoptera: Carabidae). BMC Evolutionary Biology, 13: 248-263.

Gestro R., 1900 - Gli anoftalmi trovati finora nel Veneto. Annali del Museo Civico di Storia Naturale di Genova, 40: 567-572.

Gestro R., 1907 - Una gita in Garfagnana. Annali del Museo Civico di Storia Naturale di Genova, serie 3a, Vol. 3 (43): $168-177$.

MÜLlER G., 1913 - Drei neue blinde Trechen aus Oesterreich. Entomologische Blätter, IX: 299-303.

Panajotti S., Pandolfo L., Voltan R., 1979 - Le Grotte dei Colli Euganei. Supplemento al "Notiziario" della sezione di Padova del Club Alpino Italiano, 1: 1-12.

PACE R., 1974 - Descrizione di un nuovo Orotrechus G. Müller dei Colli Euganei (Coleoptera Trechidae). Memorie del Museo Civico di Storia Naturale di Verona, 20 (1972): 495-501.

PIVA E., 2014 - Due nuovi Orotrechus Müller del Vicentino (Veneto, Italia) e note sinonimiche (Coleoptera Carabidae Trechinae). Bollettino della Società entomologica italiana, 146 (3): 113-127.

Pomini F.P., 1940 - Una nuova specie di Orotrechus (O. Juccii) delle Prealpi Veronesi (Coleopt. Carabidae). Bollettino della Società entomologica italiana, 72 (5-6): 81-86.

Vigna Taglianti A., 1981 - Un nuovo Orotrechus delle Prealpi Venete (Coleoptera, Carabidae). Bollettino del Museo Civico di Storia Naturale di Verona, 7 (1980): 69-84.

Vigna Taglianti A., 1982 - Le attuali conoscenze sui Coleotteri Carabidi cavernicoli italiani. Lavori della Società Italiana di Biogeografia, nuova serie, Vol. VII (1978): 339-430. 